\title{
Promoção de pesquisas de alta qualidade sobre as prioridades em saúde na América Latina e no Caribe*
}

\author{
Fabio Zicker ${ }^{1}$, Luis Gabriel Cuervo ${ }^{2}$ e Luis Alejandro Salicrup ${ }^{3}$
}

\author{
A Região ainda precisa fortalecer sua capacidade de pesquisa para informar políticas e \\ práticas de saúde pública, afirmam Fabio Zicker e cols.
}

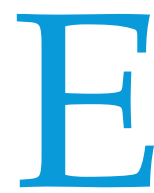
vidências científicas provenientes de pesquisas de alta qualidade são importantes para melhorar a saúde e a equidade na saúde no nível globo. ${ }^{1}$ Quando os recursos são limitados, conhecimentos e ferramentas regionais relevantes são essenciais para fortalecer a efetividade dos serviços de saúde e reduzir a carga de doenças. ${ }^{2}$

A criação de sistemas nacionais de pesquisa em saúde focados nas prioridades locais na América Latina e no Caribe (ALC) pode facilitar a realização de pesquisas de alta qualidade e fortalecer a capacidade de realização de pesquisas. ${ }^{3}$ Nos últimos 15 anos, em geral o gasto com pesquisa e desenvolvimento na Região aumentou de $0,57 \%$ para $0,8 \%$ do produto interno bruto (PIB). Porém, esse nível ainda está muito aquém em relação ao nível dos investimentos feitos por países da Organização para a Cooperação e Desenvolvimento Econômico (2,5-2,8\% do PIB). ${ }^{4}$

Apesar de o número de publicações científicas em saúde passíveis de serem citadas em referências ter triplicado na $\mathrm{ALC}^{5}$ a relevância e as possibilidades,

\footnotetext{
* Tradução oficial para o português realizada pela Organização Pan-Americana da Saúde. Em caso de discrepância, prevalecerá a versão original em inglês.
}

apresentadas pelas pesquisas, de enfrentar os desafios encontrados na Região ainda precisam melhorar. A falta de coordenação entre a pesquisa acadêmica e as necessidades de saúde da Região é amplamente reconhecida. ${ }^{6} \mathrm{O}$ custeio geral voltado para a infraestrutura científica e de pesquisa tem ficado à mercê da instabilidade social, econômica e política. As subvenções dadas às pesquisas científicas tem sido um dos primeiros itens a serem cortados do orçamento governamental quando surgem pressões econômicas. ${ }^{7}$ Nesse contexto, algumas iniciativas foram implementadas para fortalecer a capacidade e relevância da pesquisa em saúde na Região. Neste artigo, fazemos uma análise dos sucessos, das barreiras e das lições tiradas de algumas dessas iniciativas.

\section{FORTALECIMENTO DOS SISTEMAS NACIONAIS DE PESQUISA EM SAÚDE}

O crescente reconhecimento na esfera global da importância dos programas nacionais de pesquisa em saúde para

\section{MENSAGENS-CHAVE}

- Apesar da instabilidade social, econômica e política, a Região da ALC aumentou os gastos com pesquisa e desenvolvimento e triplicou o número de publicações em saúde nos últimos anos.

- O estímulo à produção de pesquisas relevantes para a saúde pública depende de processos de definição de prioridades, do fortalecimento dos sistemas nacionais de pesquisa e do melhoramento da colaboração entre a comunidade acadêmica e os responsáveis por implementar políticas de saúde.

- Pesquisas de implementação têm um importante papel no melhoramento do desempenho dos sistemas de saúde.

- Investimentos sustentáveis em infraestrutura e recursos humanos são necessários para aumentar a contribuição da pesquisa para a saúde pública.

\footnotetext{
Especialista em Ciência, Tecnologia e Inovação em Saúde Global. Centro de Desenvolvimento Tecnológico em Saúde, Fundação Oswaldo Cruz, Rio de Janeiro, Brasil. $\square$ fabio.zicker@gmail.com

2 Assessor Sênior para Promoção e Desenvolvimento de Pesquisas. Organização Pan-Americana da Saúde/Organização Mundial da Saúde, Washington, D.C., EUA
} 
melhorar os sistemas de saúde levou o Conselho de Pesquisa em Saúde para o Desenvolvimento [Council for Health Research for Development] e a Organização Pan-Americana da Saúde (OPAS) a realizarem, em 2008, um levantamento sobre os sistemas de pesquisa em 14 países da Região. Na época, apenas seis países dispunham de estruturas administrativas e de gestão implantadas, conduzidas pelo ministério da saúde ou ministério de ciência e tecnologia, e somente nove países informaram ter estabelecido prioridades para a pesquisa nacional em saúde. ${ }^{8}$ Os resultados foram discutidos durante a Primeira Conferência Latino-Americana sobre Pesquisa e Inovação em Saúde e em 2009 influenciaram a Política de pesquisa para a saúde, da OPAS. $^{3}$

Um dos principais objetivos da política foi estabelecer prioridades nacionais de pesquisa em saúde. Uma revisão feita em 2014, cinco anos após a criação da política, demonstrou ter havido um aumento no número de países da ALC que contam com uma pauta de pesquisas. Dos 37 países incluídos na revisão, 14 contavam com uma pauta de pesquisas, sendo que havia uma grande variação em termos de objetivos, conteúdo, métodos e áreas estratégicas. ${ }^{9} \mathrm{O}$ estudo destacou a necessidade contínua de implementação, pelos ministérios da saúde e dos parceiros da Região, de exercícios de definição de prioridades nacionais usando métodos sistemáticos.

\section{ALINHAMENTO DAS PRIORIDADES DE PESQUISA}

Ministérios de saúde e de ciência e tecnologia na ALC têm aumentado de forma regular o engajamento com os sistemas de atenção à saúde por meio da definição de prioridades e da promoção de pesquisas relevantes em saúde. Iniciativas de pesquisa como a EVIPnet (Rede de Políticas Informadas por Evidências $)^{10}$ e SORT IT (um programa operacional de pesquisa e capacitação) ${ }^{11}$ foram estabelecidas dentro dos ministérios da saúde de 10 países. A OPAS e a OMS facilitaram a qualificação, a criação de sites e a introdução de métodos para avaliar as evidências científicas. O objetivo desses programas é empoderar formuladores de políticas, pesquisadores e os responsáveis pela implementação, com vistas a fechar a lacuna entre a geração de conhecimentos e a aplicação desses conhecimentos nas políticas. O enfoque tem sido colocado sobre áreas como acesso a atenção de alta qualidade na área de saúde materno-infantil, prevenção e controle de doenças infecciosas, diagnóstico e tratamento da tuberculose e acesso a medicamentos de alto custo. Porém, na prática, há conflitos contínuos entre a aplicação do conhecimento atual e a geração bem como o teste de novos conhecimentos e evidências. ${ }^{3}$

Para promover a realização de pesquisas voltadas para o preenchimento de lacunas em áreas específicas do conhecimento, o Programa de Pesquisa para o Sistema Único de Saúde [Brazilian Research Program for the Unified Health System] descentralizou o custeio (US\$ 100 milhões no período de 15 anos) para pesquisa, desenvolvimento de tecnologias e inovação, emitindo chamados para a realização de pesquisas em áreas prioritárias. ${ }^{12} \mathrm{O}$ processo tem incluído exercícios de definição de prioridades no nível municipal, administrados por fundações estaduais de apoio à ciência e tecnologia, que têm incluído formuladores de políticas, pesquisadores, responsáveis pela implementação, e, em alguns casos, associações de pacientes, e que visam propor pautas de pesquisas relevantes para o nível regional. Essa estratégia se mostrou útil para aumentar a demanda por pesquisas sobre problemas prioritários, inclusive pela avaliação de tecnologias em saúde e manejo da atenção à saúde no local de atendimento - por exemplo, a realização do teste, diagnóstico e tratamento da tuberculose no mesmo centro de saúde.

\section{MELHORIA DA QUALIDADE E DOS PADRÕES DE PESQUISA}

Algumas das iniciativas mais desenvolvidas na Região para fortalecer a qualidade e os padrões de pesquisa são originárias do Caribe. Instituições acadêmicas caribenhas, em colaboração com a Rede Internacional de Epidemiologia Clínica [International Clinical Epidemiology Network] e a Colaboração Cochrane [Cochrane Collaboration], promoveram a capacitação para aprimorar a qualidade da pesquisa pública em saúde. O enfoque tem estado sobre a produção de revisões sistemáticas, os métodos de o planejamento e a avaliação de projetos, as boas práticas clínicas e a ética na condução de pesquisas. As iniciativas aproveitam programas de bolsas de estudos patrocinadas pelo Programa Líderes Emergentes para as Américas, com apoio, também, da Universidade de Ottawa, Universidade das Índias Ocidentais e da Escola Bloomberg de Saúde Pública da Universidade Johns Hopkins [Johns Hopkins Bloomberg School of Public Health.] Mais de 300 bolsistas foram capacitados até o presente. Outras iniciativas na Região, como a da Divisão do Caribe do Centro Cochrane [Cochrane Center], EUA, lançada em 2013 na Jamaica, e da Agência de Saúde Pública do Caribe estão também capacitando nas áreas de sintetização e tradução do conhecimento.

Um relatório de avaliação e impacto das atividades de capacitação no Caribe durante a década passada documentou o progresso na área de formação de equipes e no desenvolvimento de estruturas institucionais de pesquisa. ${ }^{13} \mathrm{O}$ relatório reconheceu a necessidade de promover a participação colaborativa entre pesquisadores e profissionais de saúde e otimizar tanto recursos humanos como financeiros por meio de parcerias entre instituições caribenhas e norte-americanas, bem como de iniciativas globais.

\section{FOMENTO DE LIDERANÇAS E DE UMA REDE DE CONTATOS PROFISSIONAIS}

As redes de pesquisa são uma forma eficiente de combinar diferentes disciplinas e conhecimentos para abordar problemas relevantes de saúde pública e promover pesquisas de alta qualidade. Um relatório da $\mathrm{OMS}^{14}$ identificou que colaborações exitosas dependem de que os membros da rede de contatos compartilhem objetivos comuns, da existência de uma estrutura administrativa clara, de lideranças fortes, de boas práticas de manejo, de recursos sustentados e da eficácia na comunicação.

Uma análise de redes sociais foi usada para mapear os relacionamentos entre os membros de redes de pesquisa, identificando os membros principais e os influentes. ${ }^{15}$ Uma revisão da rede de pesquisa de dengue demonstrou que a falta de homogeneidade na distribuição geográfica das atividades de pesquisa e a quase ausência de engajamento do setor privado são lacunas estratégicas importantes presentes nas iniciativas para desenvolver novas abordagens que visem enfrentar a crescente epidemia de dengue. ${ }^{16}$

A recente epidemia de microcefalia provocada pelo vírus zika no Nordeste do 
Quadro 1: Pesquisas realizadas em resposta à epidemia de síndrome de zika congênita no Brasil

- A resposta à epidemia de infecções pelo o vírus zika, em 2016, envolveu sistemas de vigilância nacional e estaduais, a comunidade científica e a OPAS. Apoio político e subsídios foram mobilizados, sendo que os provedores de serviços de saúde e o público em geral tinham grandes expectativas.

- Um Grupo de Pesquisa Interinstitucional da Epidemia de Microcefalia (Merg) elaborou e realizou um estudo de caso-controle para acessar possíveis fatores de risco associados com o aumento na incidência da microencefalia. ${ }^{17}$ Esses resultados levaram à realização de testes de infecção de zika em lactentes de 2 dias de idade para a detecção de infecção pelo vírus zika.

- As redes sociais ajudaram a alertar sobre o problema, mas também difundiram boatos, causando confusão sobre a etiologia do vírus, a transmissão pelo vetor e a possibilidade de disseminação da doença. A declaração de estado de emergência pelas autoridades brasileiras e OMS foi um ponto decisivo para mobilizar o setor da saúde pública, a sociedade civil e a comunidade acadêmica. A comunidade científica teve uma função protagonista ao descrever a síndrome congênita de zika, formular sua etiologia e juntar evidências para informar decisões sobre políticas. Essas medidas resultaram na formação da Rede Latino-Americana de Preparação para o Zika (https:/ / zikaplan.tghn.org).

Quadro 2: Melhoria da triagem do HPV na América Latina: o estudo ESTAMPA

- O ESTAMPA é conduzido pelo Centro Internacional de Investigações sobre o Câncer, da OMS, em colaboração com diversos ministérios da saúde na América Latina e com o apoio do Instituto Nacional do Câncer dos EUA, e da OPAS. O estudo está envolvendo um total de 50 mil participantes para avaliar a custo-efetividade de implementar diferentes métodos de triagem para detectar infecções por HPV.

- Realizar esse estudo de grande porte envolvendo múltiplos países está se mostrando tanto desafiador como complexo por envolver toda uma rede de cientistas, instituições e governos. A experiência tem sugerido que os melhores resultados e os dados de mais alta qualidade dependem, em grande parte, da eficácia do planejamento, do conhecimento da carga local de doença, da análise de custos para estabelecer os locais dos estudos, de uma análise do potencial de escalonamento no local, da participação comunitária e da gestão interdisciplinar.

- Dado que a pesquisa na área de implementação é um campo emergente e que as possibilidades de capacitação nas instituições acadêmicas estão ainda em fase de expansão, a OMS e o Programa Especial de Pesquisa e Ensino sobre Doenças Tropicais (TDR) criaram um curso on-line aberto, voltado para pesquisadores e para o pessoal da área de saúde responsável pela implementação. O objetivo do curso é colocar a pesquisa de implementação como uma abordagem importante para converter inovações em políticas ao facilitar o pronto acesso à capacitação nos países da ALC e em outras regiões da OMS. ${ }^{19}$

Brasil pode ensinar muito. As lideranças e a formação de redes foram essenciais para compartilhar informações, gerar e disseminar novos dados e definir linhas estratégicas de pesquisa para responder à emergência. $O$ rápido descobrimento da etiologia da doença e o desenvolvimento de rotinas de manejo foram facilitados por abordagens interdisciplinares e pela colaboração do grupo interinstitucional de pesquisa da epidemia de microcefalia (Quadro 1).

\section{PESQUISA SOBRE A IMPLEMENTAÇÃO DE PROGRAMAS}

Uma das principais mensagens veiculadas durante a segunda conferência Latino-Americana sobre pesquisa e inovação em saúde, em 2011, ${ }^{6}$ foi a de que o sucesso de uma inovação na área de pesquisa não é mensurada com base na complexidade de uma intervenção ou na solução de um problema. Em se tratando de pesquisa, a inovação pode levar a soluções simples que abordem grandes cargas econômicas e sociais sobre os sistemas de saúde. Isso é particularmente relevante no caso das pesquisas de implementação.

A área de pesquisa de implementação é uma na qual os países da ALC se mostram cada vez mais interessados. Essa pesquisa está voltada para o entendimento tanto dos determinantes do sucesso como das barreiras à introdução sustentável de estratégias e tecnologias em saúde ou ao escalonamento dessas. ${ }^{18}$ Atualmente, a pesquisa de implementação é subutilizada na Região, porém bons exemplos incluem o estudo multicêntrico ESTAMPA. O estudo foi formulado par avaliar o desempenho e a relação custo-eficácia de diferentes algoritmos e procedimentos usados na triagem do papilomavírus humano (HPV) (Quadro 2).

\section{CONCLUSÕES}

A pesquisa desempenha uma importante função no desenvolvimento sustentável ao melhorar o desempenho dos sistemas de saúde e o impacto desses sobre a saúde pública. A ciência, a tecnologia e a inovação estão incluídas em diversos objetivos de desenvolvimento sustentável das Nações Unidas. ${ }^{20}$ Os países da ALC estão comprometidos com alcançar esses objetivos, porém o crescimento da pesquisa em saúde necessário na Região ainda é heterogêneo. ${ }^{21}$ Sistemas nacionais de saúde e pesquisa precisam rever suas metas e alinhá-las, fortalecer as infraestruturas e monitorar os mecanismos para levantar dados confiáveis visando avaliar os avanços na área de desenvolvimento social.

Contribuição dos autores. Todos os autores contribuíram para o planejamento e a redação do manuscrito original. Celina Turchi e Enrique Vázquez auxiliaram com o exemplo do vírus zika, encontrado no Quadro 1, e Rolando Herrero e Maribel Almonte com o exemplo sobre a triagem do HPV, citado no Quadro 2.

Conflito de interesses. A política de conflito de interesses do British Medical Journal (BMJ) foi lida e compreendida, 
e fica declarado aqui que não há conflito de interesses. Fabio Zicker recebeu auxílio por meio dos processos No. 458100/2014-5 e No. 440231/2015-9 concedidos pelo Conselho Nacional de Desenvolvimento Científico e Tecnológico do Brasil (CNPq).

Procedência do artigo e revisão por pares. Encomendado; revisão por pares externa.
Declaração. As opiniões externadas neste manuscrito são de responsabilidade do(s) autor(es) e não refletem necessariamente os critérios e políticas da RPSP / PAJPH e/ou da OPAS/OMS.

Série Fortalecimiento da pesquisa em saúde nas Américas. Este artigo faz parte de uma série proposta pela OPAS e encomendada pelo BMJ, o qual foi responsável pela revisão por pares, edição e

\section{REFERÊNCIAS}

1. Organização Mundial da Saúde. Strategy on research for health. 2012. http:// www.who.int/phi/WHO_Strategy_on _research_for_health.pdf

2. Ghaffar A, Jsselmuiden I, Zicker F. Changing mindsets: research capacity strengthening in low- and middle-income countries. OMS, 2010. http:// www.who.int/tdr/publications/documents /changing_mindsets.pdf

3. Organização Pan-Americana da Saúde/ Organização Mundial da Saúde. D.C., Política de pesquisa para a saúde. $49^{\circ}$ Conselho Diretor, $61^{\text {a }}$ Sessão do Comitê Regional. 2009. https://www.paho.org/hq/index .php?option $=$ com_docman \&task $=$ doc _download\&gid=2634\&Itemid $=270 \&$ lang $=\mathrm{pt}$

4. Banco Mundial. Gasto en investigación y desarrollo (\% del PIB) https://datos .bancomundial.org/indicador/GB.XPD .RSDV.GD.ZS?locations $=$ XJhttps: $/ /$ data .worldbank.org/indicator/GB.XPD.RSDV

5. Scimago Journal \& Country Rank. (Consultado em 16 de outubro de 2017). http:/ / www.scimagojr.com

6. Council on Health Research for Development (COHRED). Report to the second Latin American conference on research and innovation for health, Panama City, 2011. http: / / www.cohred.org/publications /cohred-publications/joint-publications / report-to-the-2nd-latin-american -conference-on-research-and-innovation -for-health/

7. Ciocca DR, Delgado G. The reality of scientific research in Latin America; an insider's perspective. Cell Stress Chaperones 2017;22:847-52. doi:10.1007/s12192-017 $-0815-8$

8. Alger J, Becerra-Posada F, Kennedy A, Martinelli E, Cuervo LGGrupo Colaborativo de la Primera Conferencia Latinoamericana de
Investigación e Innovación para la Salud. Rev Panam Salud Publica 2009;26:447-57.

9. Becerra-Posada F, de Snyder NS, Cuervo LG, Montorzi G. Agendas de investigación priorizadas: un recurso estratégico para la salud en América Latina. Rev Panam Salud Publica 2014;36:361-7.

10. Organização Mundial da Saúde. EVIPnet. http://www.who.int/evidence/en/

11. Organização Mundial da Saúde. Special programme on research and training in tropical diseases. SORT IT.

12. Ministério da Saúde Brasil. Programa de Pesquisa para o SUS (PPSUS). 2017. http://www.brasil.gov.br/noticias/saude /2017/02/ministerio-da-saude-garante-80 -dos-recursos-para-pesquisa-no-setor-no -pais

13. Organização Pan-Americana da Saúde/ Organização Mundial da Saúde. D.C., Report on strengthening research capacities for health in the Caribbean, 2007-2017. 2017. http://iris.paho.org/xmlui/handle $/ 123456789 / 34342$

14. World Health Organization, Special Programme for Research and Training in Tropical Diseases. Key enabling factors in effective and sustainable research networks. 2016. http://www.who.int/tdr /publications/research-networks/en/

15. Fonseca BdeP, Sampaio RB, Fonseca MVA, Zicker F. Co-authorship network analysis in health research: method and potential use. Health Res Policy Syst 2016;14:80. doi:10.1186/s12961-016-0104-5

16. Fonseca BdeP, Zicker F. Dengue research networks: building evidence for policy and planning in Brazil. Health Res Policy Syst 2016;14:34. doi:10.1186/s12961-016-0104-5

17. de Araújo TVB, Rodrigues LC, de Alencar Ximenes RA, et al, pesquisadores do Grupo de Pesquisa da Epidemia de Microencefalia Ministério da Saúde decisão de publicar o artigo, sem participação da OPAS. As despesas referentes à publicação desta série em Open Access foram financiadas pela OPAS. Para ler os outros artigos da série em inglês, visite www.bmj.com/health-research -americas; acesso à série em espanhol e português em https://www.paho .org/journal/es/numeros-especiales / fortalecimiento-investigacion-para -salud-americas
Organização Pan-Americana da Saúde Instituto de Medicina Integral Professor Fernando Figueira Secretaria da Saúde Estado de Pernambuco. Association between Zika virus infection and microcephaly in Brazil, January to May, 2016: preliminary report of a case-control study. Lancet Infect Dis 2016;16:1356-63. doi:10 .1016/S1473-3099(16)30318-8

18. Peters DH, Adam $\mathrm{T}$, Alonge $\mathrm{O}$, Agyepong IA, Tran N. Implementation research: what it is and how to do it. BMJ 2013;347:f6753. doi:10.1136/bmj.f6753.

19. Organização Mundial da Saúde, Special Programme for Research and Training in Tropical Diseases. New online course on implementation research under development. TDR News 2015. http://www.who .int/tdr/news/2015/new-online-course /en/

20. Nações Unidas. Objetivos de desenvolvimento sustentável: 17 objetivos para transformar nosso mundo. https:// www.unric.org/pt/17-objetivos-de -desenvolvimento-sustentavel

21. Becerra-Posada F, Minayo M, Quental C, de Haan S. National research for health systems in Latin America and the Caribbean: moving towards the right direction? Health Res Policy Syst 2014;12:13. doi:10 $.1186 / 1478-4505-12-13$

Como citar (artigo original): BMJ 2018;362:k2492 http:/ / dx.doi.org/10.1136/bmj.k2492

Manuscrito original em inglês publicado em 16 de julho de 2018 . 